Wright State University

CORE Scholar

\title{
Impact of Spatial Variability and Masker Fringe on the Detectability of a Brief Signal
}

\author{
Michelle H. Wang \\ Wright State University
}

Follow this and additional works at: https://corescholar.libraries.wright.edu/etd_all

Part of the Industrial and Organizational Psychology Commons

\section{Repository Citation}

Wang, Michelle H., "Impact of Spatial Variability and Masker Fringe on the Detectability of a Brief Signal" (2019). Browse all Theses and Dissertations. 2265.

https://corescholar.libraries.wright.edu/etd_all/2265

This Thesis is brought to you for free and open access by the Theses and Dissertations at CORE Scholar. It has been accepted for inclusion in Browse all Theses and Dissertations by an authorized administrator of CORE Scholar. For more information, please contact library-corescholar@wright.edu. 


\title{
IMPACT OF SPATIAL VARIABILITY AND MASKER FRINGE ON THE DETECTION OF A BRIEF SIGNAL
}

\author{
A thesis submitted in partial fulfillment \\ of the requirements for the degree of \\ Master of Science
}

by

MICHELLE H. WANG

B.S., Otterbein University, 2015

2019

Wright State University 
Wright State University

GRADUATE SCHOOL

September 20, 2019

I HEREBY RECOMMEND THAT THE THESIS PREPARED UNDER MY

SUPERVISION BY Michelle H. Wang ENTITLED Impact of Spatial Variability and Masker Fringe on the Detectability of a Brief Signal BE ACCEPTED IN PARTIAL FULFILLMENT OF THE REQUIREMENTS FOR THE DEGREE OF Master of Science.

Robert H. Gilkey

Thesis Director

Debra Steel-Johnson

Department Chair

Committee

Brian D. Simpson, Ph.D.

Scott N. J. Watamaniuk, Ph.D.

Barry Milligan, Ph.D.

Interim Dean, Graduate School 


\begin{abstract}
Wang, Michelle H. MS., Department of Psychology, Wright State University, 2019. Impact of Spatial Variability and Masker Fringe on the Detectability of a Brief Signal.
\end{abstract}

The effect of masker spatial variability and masker fringe on the perception of a brief tone in noise was investigated in a detection task. Simpson (2011) found large effects of spatial variability (randomizing masker locations from trial to trial) in a masked localization experiment, as well as two effects of masker fringe (masking noise before the onset of the target): 1) cuing the masker location (spatial cuing effect) and 2) temporally separating the onset of the masker and the onset of the target (onset effect). In contrast, in detection studies, the effects of masker spatial variability are small (e.g., Bernstein \& Trahiotis, 1997) and the possibility of a spatial cuing effect has not been directly examined. However, onset effects of similar magnitude to those observed by Simpson have been reported (e.g., McFadden, 1966). To determine whether these differences in the effect of masker variability between localization and detection could be attributed to the fact that in localization experiments there is also trial-to-trial variability in the target, we conducted a detection experiment via headphones using a 2 Masker Variability (variable \& fixed) x 2 Target Variability (variable \& fixed) x 2 Masker Fringe (no fringe $\&$ fringe) factorial design. We used a $60-\mathrm{ms}, 500-\mathrm{Hz}$ sinusoidal target and a $60-\mathrm{ms}$ Gaussian noise masker (and a 500-ms Gaussian noise masker fringe in the fringe conditions). Masker and/or target location was varied laterally by varying the interaural 
time difference (ITD) of the fine structure. We found little effect of masker variability, in agreement with previous detection studies, and the presence or absence of target spatial variability did not alter the pattern of results. Because the effect of masker variability was small, there was limited opportunity to observe a spatial cuing effect, but there was an onset effect of fringe that was of similar magnitude to that observed in previous detection studies. In the binaural detection literature, the onset effect is large under dichotic conditions than dichotic conditions. Similarly, the onset effect we observed varied significantly with the spatial separation between the target and the masker, being small when the target and masker were collocated and large when they were widely separated. Various explanations for the results were considered. Spatial transition appears to be a reasonable explanation for the detection results, but not for localization; and segregation appears to be a reasonable explanation for the localization results, but not for detection. Although the data in the literature on the overshoot effect are somewhat inconsistent, it seems possible that overshoot could be used to explain both sets of data. 


\section{TABLE OF CONTENTS}

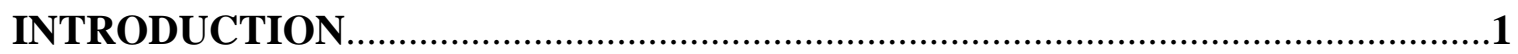

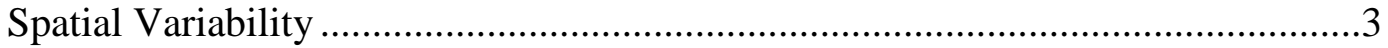

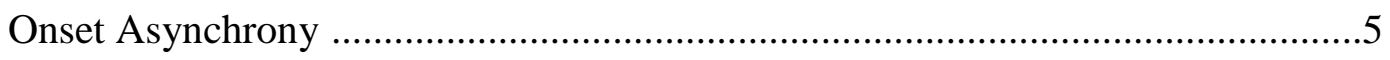

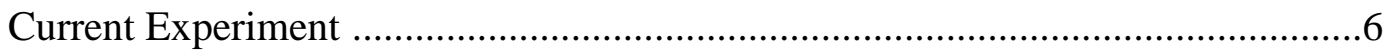

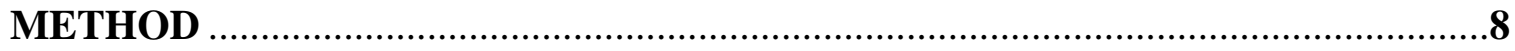

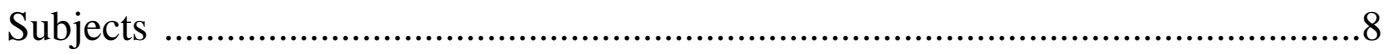

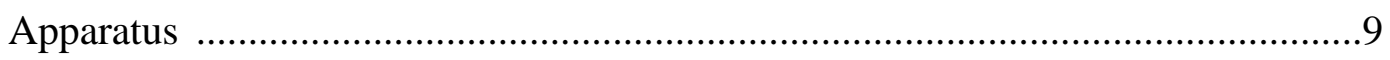

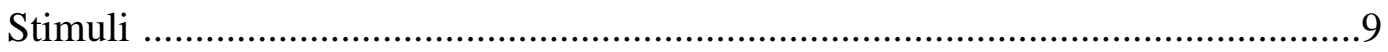

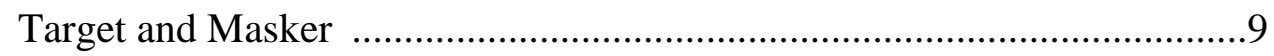

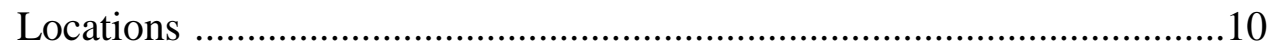

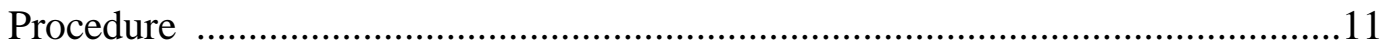

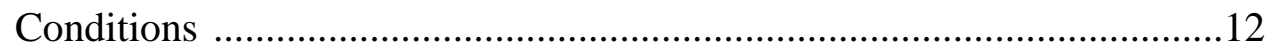

Preliminary Experiment .......................................................................14

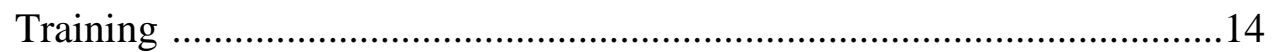

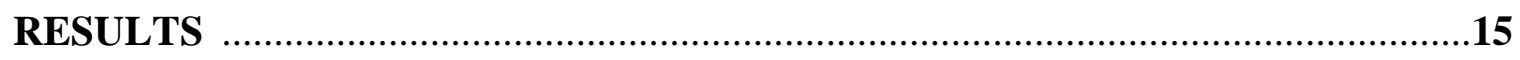

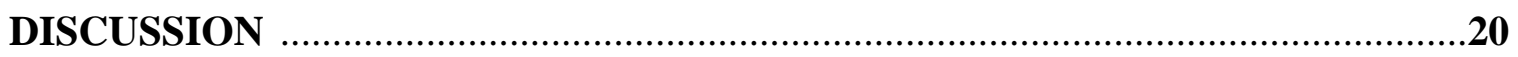

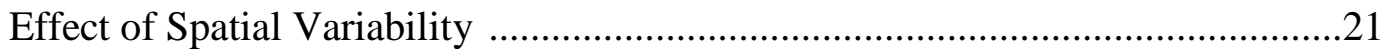

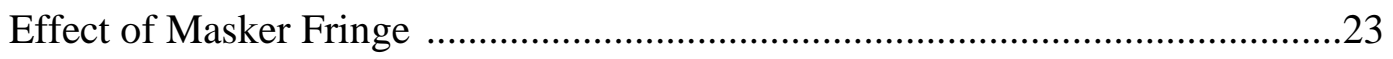


Conclusion

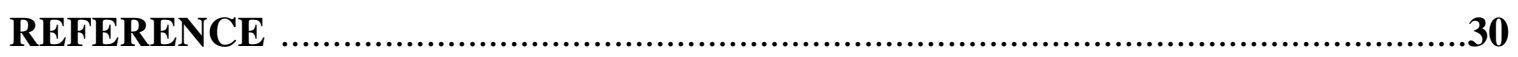

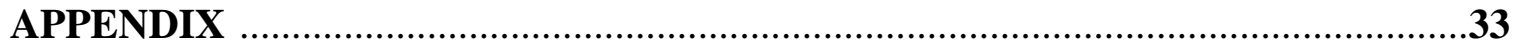




\section{LIST OF FIGURES}

1. Masker configurations in Simpson (2011) ........................................................ 2

2. Threshold plotted for the various Spatial Variability conditions in both Masker

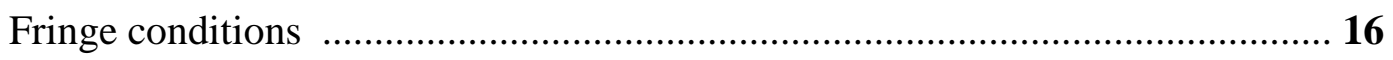

3. Threshold in the $\mathrm{M}_{\mathrm{F}} \mathrm{T}_{\mathrm{F}}$ condition plotted as a function of ITD Difference ........... 18

A.1. Measured thresholds for 12 selected $\mathrm{M}_{\mathrm{F}} \mathrm{T}_{\mathrm{F}}$ configuration in the preliminary

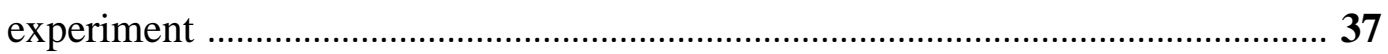

A.2. MLD estimates for configurations in which the target and masker are collocated or that are "symmetric", about the median plane, to measured configurations ... 38

A.3. MLD estimates for configurations that differ by one or two spatial position from "known" configurations.

A.4. MLD estimates calculated from four known masker/target configurations that shared the same target location and a masker location that differed by one spatial position or the same masker location and a target location that differed by one spatial position

A.5. MLD estimates calculated from four known configurations that shared the same target location and a masker location that differed by one or two spatial position or the same masker location and a target location that differed by one or two spatial positions 


\section{LIST OF TABLES}

1. Equally-spaced locations from the left side of the head to the right side of the head with their corresponding Interaural Time Delays (ITDs)

2. SIAM Transition Matrix

3. 3-Way ANOVA Results for Target Variability, Masker Variability, and Masker

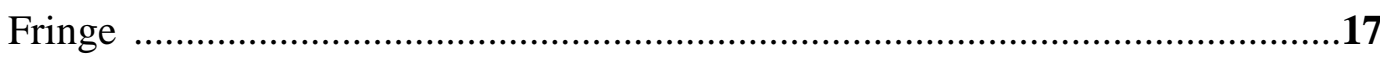

4. Differences in ITDs between masker and target for each MFTF configuration ... 19 
Impact of Spatial Variability and Masker Fringe on the Detectability of a Brief Signal

Simpson (2011) was interested in the impact of spatial variability and onset asynchrony on the localization of a wideband, click-train target in a wideband noise masker. He found that when the masker and target were pulsed on and off together (the "pulsed" condition, Figure 1a), randomizing the location of the masker from trial to trial reduced target localization accuracy in the Left/Right dimension, such that the signal-tonoise ratio (SNR) had to be increased by $10.0 \mathrm{~dB}$ to achieve localization performance equivalent to the case in which the location of the masker was fixed and known throughout a block of trials. Similar but smaller effects were observed in the Front/Back and $\mathrm{Up} /$ Down dimensions, $3.3 \mathrm{~dB}$ and $4.6 \mathrm{~dB}$, respectively. Simpson argued that spatial variability of the masker made it more difficult to separate it perceptually from the target, so that target-localization errors increased. This effect of masker spatial variability was largely mitigated (SNR could be lowered by $7.8 \mathrm{~dB}$ ) when a preview of the masker location was provided $500 \mathrm{~ms}$ before the presentation of the masker and target (the "Cue" condition, Figure 1b). Performance was further improved in both the variable masker and fixed masker conditions (by $4.8 \mathrm{~dB}$, on average) when the preview was presented immediately before the masker and the target, that is, without the 500-ms gap (the "Fringe" condition, Figure 1c). Simpson argued that the 500-ms onset asynchrony between the noise and the target in this fringe condition temporally separated the activity associated with the onset of the target from the activity associated with the onset of the masker, making it easier to detect the target and/or perceptually segregate it from the 
masker. And so, Simpson concluded that a masker fringe has two effects: 1) the spatial cuing effect - reducing effects of masker spatial variability, and 2) the onset effect temporally separating the masker and target onsets.

Similar questions regarding spatial variability and masker fringe have been examined in detection experiments. In fact, Simpson's (2011) study was motivated, in part, by the binaural detection literature on masker fringe (e.g., McFadden, 1966; Yost 1985).

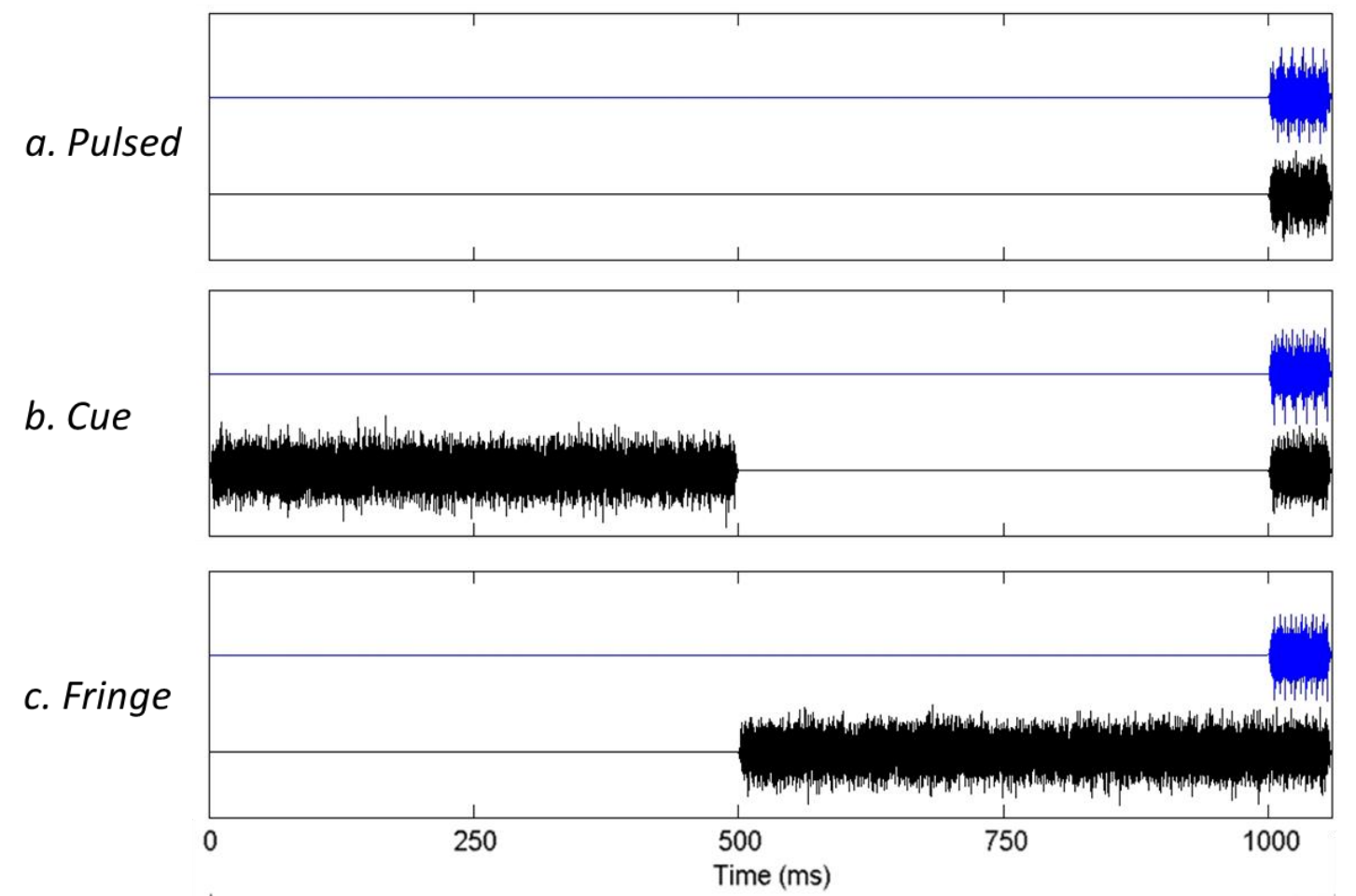

Figure 1. Masker configurations in Simpson (2011). In each panel, the upper trace shows the target, and the lower trace shows the cue, fringe, and masker stimuli. Panel a) depicts the "pulsed" condition, in which the masker and target pulsed on an off together. Panel b) depicts the "Cue" condition, in which a 500-ms preview of the masker was presented 500 
ms before the masker and target (Simpson referred to this as the "Gap" condition). Panel c) depicts the "Fringe" condition, in which a 500-ms preview of the masker was presented immediately before the masker and target.

\section{Spatial Variability}

Simpson (2011) reported a large effect of randomizing masker location from trial to trial (i.e., spatial variability). However, the few detection studies that have examined spatial variability have found much smaller effects. For example, Fan, Streeter, and Durlach (2008) examined the impact of masker spatial variability on target detectability using a set of three simultaneous, dichotic, narrowband, noise maskers and a diotic, simultaneous, narrowband, noise target (statistically identical to the maskers). Masker locations were either fixed and known throughout a block of trials or were randomized from trial to trial by selecting 1 of 10 sets of triplet maskers (note, each set included three different interaural time delays [ITDs; one for each masker], which were selected without replacement from a set of eight ITDs). Masker ITDs were never within $\pm 200 \mu$ s of the target ITD (i.e., $0 \mu \mathrm{s}$ ), thus forming a "protected zone" around the target. They observed a 0.7-dB effect of masker spatial variability. Bernstein and Trahiotis (1997) examined the impact of spatial variability on the detectability of a 500-Hz pure tone target in broadband noise. Spatial variability was created by randomizing the ITD of the entire masker-plustarget waveform (i.e., the "distance" between the masker and the target remained constant from trial to trial). They found that the impact of spatial variability on target detectability 
was small (but statistically significant), $1.5 \mathrm{~dB}$ with a $25-\mathrm{ms}$ target and a $35-\mathrm{ms}$ masker and $2.7-\mathrm{dB}$ with a $125-\mathrm{ms}$ target and a $145-\mathrm{ms}$ masker.

Although these studies used different stimuli and designs, they yielded similar low estimates of the impact of spatial variability. These estimates are much smaller than the 10-dB effect observed in Simpson's (2011) localization study. This could simply indicate that the impact of spatial variability is different for localization and detection or may be due to other methodological differences among the studies (e.g., target duration, target bandwidth, and target spatial variability) as discussed below.

The detection studies examining spatial variability did not directly consider the impact of masker fringe. In Fan et al. (2008), the masker and target were turned on and off together (i.e., no masker fringe). Bernstein and Trahiotis (1997) used brief (5 ms and $10 \mathrm{~ms}$ ) forward (immediately preceding the target) and backward (immediately following the target) fringes, which could possibly have functioned as spatial cues to the location of the masker. The small effects of spatial variability were slightly less (by about $1 \mathrm{~dB}$ ) with 10-ms fringes relative to 5-ms fringes. However, fringe duration and target duration were confounded (i.e., the $10 \mathrm{~ms}$ fringe was always paired with the $125 \mathrm{~ms}$ target duration and the $5 \mathrm{~ms}$ fringe was always paired with the $25 \mathrm{~ms}$ target duration). So, because the effect of spatial variability was small in these studies $(0.7-2.7 \mathrm{~dB})$ and the presence of masker fringe was not systematically varied, it is difficult to know with certainty whether masker fringe could serve as a spatial cue and mitigate these small effects of masker spatial 
variability. Nevertheless, it is reasonable to conclude that any spatial cuing effect is likely much smaller than the 7.8-dB effect reported by Simpson (2011).

\section{Onset Asynchrony}

Simpson (2011) found that performance was better in the fringe condition relative to the Pulsed condition ( $4.8 \mathrm{~dB})$ even when the masker location was fixed and known throughout a block of trials. That is, there appeared to be a benefit of the masker fringe beyond cuing the location of the masker. He referred to this additional benefit of the masker fringe as the "onset effect," noting the fringe acted to separate temporally the onset of the masker from the onset of the target. Two areas of research within the detection literature seem related to the onset effects that Simpson observed, research on masker fringe and research on temporal masking. Masker fringe studies compare thresholds between pulsed and fringe conditions similar to those of Simpson's. Temporal masking studies compare conditions in which the target is presented near the beginning of the masker (as with the Pulsed condition, but typically with a "fringe" following the masker as well) to conditions in which the target is, for example, presented near the end of the masker (similar to the Fringe condition). However, the finding from these areas are somewhat contradictory. Binaural detection research on the impact of masker fringe has routinely shown a binaural fringe effect of 5-9 dB (i.e., detectability increased with the addition of the fringe) under dichotic conditions, but little fringe effect under the diotic conditions (e.g., McFadden, 1966; Robinson \& Trahiotis, 1972; Yost, 1985). On the other hand, the seemingly closely related temporal masking literature has shown a 3- to 5-dB 
effect of delaying target onset (i.e., increase in detectability when the target was presented near the end of the masker rather than near the beginning of the masker) under monaural or diotic conditions (Zwicker, 1965; McFadden, 1988; Simpson, 1995). But, the findings in the literature for dichotic conditions are somewhat mixed. McFadden (1988) observed a negligible effect of delaying target onset under dichotic condition, whereas Simpson (1995) observed about a 4-dB effect.

\section{Current Experiment}

The effects of masker spatial variability observed in the detection literature are small compared to the effects that Simpson (2011) observed in a masked localization task. There are a number of possible explanations for the observed difference: 1) In a localization task, even when the location of the masker is fixed, the location of the target still varies from trial to trial. That is, the localization task "requires" a certain level of spatial variability. Perhaps, masker spatial variability and target variability interact in such a way that the effects of masker spatial variability are much larger when target spatial variability is also present. Detection studies examining other stimulus parameters have observed a small interaction between masker and target variability. Spiegel and Green (1982) found that the effect of masker waveform variability was greater when the frequency of the target was also randomized from trial to trial (4-dB effect of masker variability) relative to when it was fixed and known throughout a block of trials (3-dB effect of masker variability). 2) In the detection studies considered here, the target is low frequency and narrowband, whereas Simpson used a wideband target. Moreover, 
Simpson's study was conducted in the free field, whereas these detection studies presented sounds through headphones (Fan et al., 2008, filtered with head-related transfer functions in some conditions). It is possible that information other than the ITDs typically varied in detection studies was the basis of listeners localization judgments in Simpson. Perhaps interaural level differences (ILDs) and direction dependent spectral shape information in higher frequency regions or the comparison of information across frequency regions is more easily disrupted by masker spatial variability. However, this seems unlikely given that Simpson found the largest effects in the Left/Right dimension and Left/Right judgements are typically thought to be dominated by low-frequency ITDs (Wightman and Kistler, 1992). 3) Localization requires a spatial judgment, whereas detection does not necessarily. Perhaps this spatial judgment, or the underlying processing supporting it, is more easily disrupted by spatial variability.

The current study focuses on the first of these possibilities, that is, the impact of target variability. In addition, we examined whether separable spatial cuing and onset effects of masker fringe can be observed in a detection task. We manipulated Masker Fringe, Masker Variability, and Target Variability in a factorial design. We expected that when the target location was variable, we would observe effects of Masker Fringe and Masker Variability similar to those observed by Simpson (2011; i.e., a large effect of Masker Variability and both effects of Masker Fringe - the onset effect and the spatial cuing effect). We expected that when the target location was fixed, we would observe effects of Masker Fringe and Masker Variability similar to those observed in the 
detection literature (i.e., little effect of Masker Variability and a 5- to 9-dB effect of Masker Fringe - that is, an onset effect of fringe, but not the spatial cuing effect). These predictions correspond to interactions between Target Variability and Masker Variability and between Target Variability and Masker Fringe. Finally, consistent with the binaural detection literature, we expected that the onset effect of fringe would be small under diotic conditions and increase as a function of spatial separation between the masker and the target (i.e., an interaction between masker fringe and spatial separation).

\section{Method}

We measured detection thresholds in a 2 (Masker Fringe) x 2 (Masker Spatial Variability) x 2 (Target Spatial Variability) factorial design. The two Masker Fringe conditions examined were: 1) the masker was pulsed on and off with the target (Pulsed condition; Fig. 1a), and 2) the onset of the masker occurred $500 \mathrm{~ms}$ prior to the onset of the target and the stimuli were gated off together (Fringe condition; Fig. 1c). The two Masker Spatial Variability conditions examined were: 1) the masker location was fixed throughout a block of trials $\left(\mathrm{M}_{\mathrm{F}}\right)$, and 2) the masker location was varied randomly from trial to trial $\left(\mathrm{Mv}_{\mathrm{v}}\right)$. Similarly, the two Target Spatial Variability conditions examined were target location fixed $\left(\mathrm{T}_{\mathrm{F}}\right)$ and variable $\left(\mathrm{T}_{\mathrm{V}}\right)$.

\section{Subjects}

The current study employed 5 listeners (3 females and 2 males), who were 22-33 years-old $(M=27, S D=3.74)$. All listeners were tested to ensure that they had normal hearing (audiometric threshold of $15 \mathrm{~dB}$ HL or better between $125 \mathrm{~Hz}$ and $8 \mathrm{kHz}$ ). Four 
of the listeners were chosen from members of a subject panel at Wright Patterson Air Force Base, which is composed of long-term, part-time listeners compensated at an hourly rate. The fifth listener was the author. All listeners had previously participated in sound localization and/or detection experiments.

\section{Apparatus}

Listeners were seated in individual Industrial Acoustics Company soundattenuating booths. Each booth was equipped with a Dell Precision Workstation T1700 computer with Matlab software, a Fireface UCX audio card, and a pair of Sennheiser HMD 280-13 headphones (300 $\Omega$ ) to administer the task and deliver the stimuli. Two stations with the same system configuration (booth, computer, sound card, and headphones) were used to conduct the experiment. Both systems were calibrated with a $500-\mathrm{Hz}$ pure tone (target frequency) to ensure the output levels in all headphones were equal.

\section{Stimuli}

Target and masker. The target was a $500-\mathrm{Hz}$ pure tone with a duration of $60 \mathrm{~ms}$, including 5-ms on/off ramps (Matlab BLACKMAN function). On each trial, we generated a 560-ms Gaussian noise (Matlab RANDN function) at a sample rate of $50 \mathrm{kHz}$. The noise was up-sampled by a factor of 20 (Matlab RESAMPLE function), allowing interaural delays to be introduced with $1-\mu$ s resolution, down-sampled to the original sample rate, and then filtered using a $4^{\text {th }}$-order bandpass Butterworth filter with cutoff frequencies of $100 \mathrm{~Hz}$ and $3000 \mathrm{~Hz}$ (Matlab BUTTER and FILTER functions). In the Pulsed condition, 
the generated noise was windowed to have a duration of $60 \mathrm{~ms}$, including 5-ms on/off ramps and was temporally aligned so that its onset and offset were synchronous with those of the target. In the fringe condition, the noise was windowed to have a duration of $560 \mathrm{~ms}$, including 5-ms on/off ramps, and was temporally aligned so that its onset occurred $500 \mathrm{~ms}$ before that of the target and its offset was synchronous with that of the target. The interaural time delays (ITDs) and windowing were implemented such that the delays were present in the fine-structure of the target with no interaural onset or offset delays.

Locations. The spatial locations of the stimuli were manipulated by applying one of 13 ITDs (see Table 1), which were chosen to create spatial images that were approximately equally spaced perceptually and spanned the interaural axis within the head, based on Yost's (1981) lateralization data at $500 \mathrm{~Hz}$. The ITD of the masker fringe, if present, was always the same as the ITD of the following masker. For clarity, each location is referred to by its corresponding ITDs in positive (right side of head) or negative (left side of head) microseconds $(\mu \mathrm{s})$, and a specific masker and target location configuration is referred to by its masker and target ITDs (e.g., $\mathrm{M}_{-667} \mathrm{~T}_{112}$ ).

Table 1

Equally-spaced locations from the left side of the head to the right side of the head with their corresponding Interaural Time Delays (ITDs)

\begin{tabular}{cccccccccccccc}
\hline Locations & 1 & 2 & 3 & 4 & 5 & 6 & 7 & 8 & 9 & 10 & 11 & 12 & 13 \\
ITDs $(\mu \mathrm{s})$ & -667 & -494 & -309 & -182 & -112 & -56 & 0 & 56 & 112 & 182 & 309 & 494 & 667 \\
\hline
\end{tabular}

Note. ITDs are based on Yost's (1981) lateralization data at $500 \mathrm{~Hz}$. 


\section{Procedure}

A single-interval Yes/No task was employed. Each trial began with a 100-ms green warning signal, followed by a $150-\mathrm{ms}$ pause, and then by a $560-\mathrm{ms}$ observation interval (the masker, and the target if present, occurred during the final $60 \mathrm{~ms}$ of the observation interval; the first $500 \mathrm{~ms}$ of the observation interval was silent under the Pulsed conditions and contained the masker fringe under the fringe condition). After the observation interval the listeners responded at their own pace by pressing the " $\mathrm{C}$ " key on the keyboard for "Yes," if they believed the target had been presented, or the "M" key for "No." The a priori probability of the target being presented was 0.5 . After the response, correct-answer feedback was provided for $560 \mathrm{~ms}$, which was followed by a $250 \mathrm{~ms}$ inter-trial interval before the next trial began.

We used the Single-Interval Adjustment Matrix (SIAM) adaptive staircase procedure to estimate the $75 \%$ correct threshold for each block (Kaernbach, 1990). Before each block began, repeating sample trials that alternated between target present and target absent were presented (same experimental condition as the current block). These sample trials served to familiarize the listener with the stimuli for the block. Listeners did not respond to the sample trials. The listener could listen to an unlimited number of sample trials and pressed the "C" or " $\mathrm{M}$ " key on the keyboard to begin the staircase procedure. The starting level of the target was $8 \mathrm{~dB}$ above the expected threshold (based on the results of the preliminary experiment described below) and was adjusted after each trial as indicated by the SIAM transition matrix (Table 2). SIAM 
adjustment levels were multiplied by a step size and added to the current target level to determine the target level for the next trial. The initial step size was $2 \mathrm{~dB}$, which was reduced to $1 \mathrm{~dB}$ after the second reversal. Each block ended after 14 reversals and the threshold was computed as the average of the levels that led to the last 10 reversals.

A block took about 3 minutes to complete. Short breaks were administered between blocks ( 45 seconds after every block; at least 5 minutes after every $4^{\text {th }}$ block). Listeners completed either one 30-minute session or one 60-minute session per business day depending on their availability. There was a two-week break due to holidays, during which no sessions were conducted.

Table 2

SIAM Transition Matrix

\begin{tabular}{r|c|c|}
\multicolumn{1}{c}{ “Yes" } & "No" \\
\cline { 2 - 3 } Target + Masker & -1 & +1 \\
\cline { 2 - 3 } Masker Only & +2 & +0 \\
\cline { 2 - 3 } & &
\end{tabular}

Note. With these values SIAM estimates the $75 \%$ correct point on the psychometric function.

\section{Conditions}

Within a block of trials, the masker was either fixed (presented from the same location on each trial) or variable (presented from a different, randomly-selected location on each trial); similarly, the target could be fixed or variable. So, there were four possible 
levels of spatial variability: Fixed Masker/Fixed Target $\left(\mathrm{M}_{\mathrm{F}} \mathrm{T}_{\mathrm{F}}\right)$, Fixed Masker/Variable Target $\left(\mathrm{M}_{\mathrm{F}} \mathrm{T}_{\mathrm{V}}\right)$, Variable Masker/Fixed Target $\left(\mathrm{Mv}_{\mathrm{V}} \mathrm{T}_{\mathrm{F}}\right)$, and Variable Masker/Variable Target $\left(M_{v} T_{v}\right)$. The Masker Fringe condition (pulsed and fringe) was also held constant within a block. In the $\mathrm{M}_{\mathrm{F}} \mathrm{T}_{\mathrm{F}}$ conditions, thresholds were calculated as the average of 2 adaptive tracks for each of 12 selected masker/target spatial configurations (the procedure used to select the 12 configuration is described in the Appendix). In addition, we measured thresholds for the $\mathrm{M}_{0} \mathrm{~T}_{1000}$ spatial configuration (i.e., the $\mathrm{N}_{0} \mathrm{~S}_{\pi}$ configuration), allowing direct comparison to equivalent conditions in the binaural detection literature. So, a total of 26 blocks were conducted for each listener under the $\mathrm{M}_{\mathrm{F}} \mathrm{T}_{\mathrm{F}}$ condition $([12+1]$ target/masker configurations $\mathrm{x} 2$ tracks) for both the pulsed and fringe conditions. In the $\mathrm{M}_{\mathrm{F}} \mathrm{T}_{\mathrm{V}}$ conditions, a fixed masker location was selected from 5 possible locations at the beginning of the block ( $\mathrm{M}_{-667}, \mathrm{M}_{-182}, \mathrm{M}_{0}, \mathrm{M}_{182}$, or $\left.\mathrm{M}_{667}\right)$, and the target location was randomly selected on each trial from the set of 13 possible target locations. A total of 20 blocks were conducted for each listener under the $\mathrm{M}_{\mathrm{F}} \mathrm{T}_{\mathrm{V}}$ condition (5 masker locations $\mathrm{x} 4$ tracks) for both the pulsed and fringe conditions. In the $\mathrm{MV}_{\mathrm{F}}$ conditions, a fixed target location was selected from 5 possible target locations at the beginning of the block ( $\mathrm{T}_{-667}, \mathrm{~T}_{-182}, \mathrm{~T}_{0}, \mathrm{~T}_{182}$, or $\left.\mathrm{T}_{667}\right)$, and the masker location was randomly selected on each trial from the set of 13 possible masker locations. A total of 20 blocks were conducted for each listener under the $\mathrm{MVT}_{\mathrm{F}}$ condition (5 target locations $\mathrm{x} 4$ tracks) for both the pulsed and fringe conditions. In the $\mathrm{M}_{\mathrm{V}} \mathrm{T}_{\mathrm{V}}$ condition, masker and target locations were independently and randomly selected on each trial from the set of 
13 possible locations. A total of 20 blocks were conducted for each listener under the $M_{v} T_{v}$ condition for both the pulsed and fringe conditions.

Preliminary experiment. In the experiment proper, under the variable conditions the location of the masker $\left(\mathrm{MvT}_{\mathrm{F}}\right)$, the location of the target $\left(\mathrm{M}_{\mathrm{F}} \mathrm{T}_{\mathrm{V}}\right)$, or both $\left(\mathrm{MV}_{\mathrm{V}}\right)$ were randomly selected on each trial. So, the difference between the ITDs for the masker and the target also varied randomly from trial to trial. These variations in ITD difference would normally lead to substantial variation in detectability due to the binaural masking level difference (MLD). Because our focus was on the impact of variation in spatial location, rather than the impact of variation in detectability, we adjusted the level of the target on each trial to compensate for the estimated MLD for the particular ITD difference presented, thereby keeping detectability approximately constant and equal to the detectability for the $\mathrm{M}_{0} \mathrm{~T}_{0}$ configuration (obviously, detectability still changed as governed by the SIAM adaptive staircase procedure).

In a preliminary experiment, we measured thresholds for 13 (the 12 selected $\mathrm{M}_{\mathrm{F}} \mathrm{T}_{\mathrm{F}}$ configurations and $\left.\mathrm{M}_{0} \mathrm{~T}_{1000}\right)$ target/masker spatial configurations and used them to estimate the MLD for each of the 169 possible masker/target spatial configurations. This was done individually for each listener in both Masker Fringe conditions. The estimation procedure is described in the Appendix.

Training. Listeners received training at three points during the study: 1) before the preliminary experiment, 2) after the two-week holiday break, which occurred during the preliminary experiment, and 3) before the main experiment. At the first two time 
points, listeners completed 2 tracks of each of the 12 selected configurations plus the $\mathrm{M}_{0} \mathrm{~T}_{1000}$ configuration under the $\mathrm{M}_{\mathrm{F}} \mathrm{T}_{\mathrm{F}}$ condition in both Masker Fringe conditions.

Listeners were not trained in the variable conditions because those conditions were not included in the preliminary study. At the third point in time, listeners completed 2 tracks of each configuration under the $\mathrm{M}_{\mathrm{F}} \mathrm{T}_{\mathrm{V}}$ condition (5 fixed masker locations $\left[\mathrm{M}_{-667}, \mathrm{M}_{-182}\right.$, $\left.\mathrm{M}_{0}, \mathrm{M}_{182}, \mathrm{M}_{667}\right]$ with randomized target locations), 2 tracks of each configuration under the $\mathrm{M}_{\mathrm{V}} \mathrm{T}_{\mathrm{F}}$ condition (5 fixed target locations [ $\mathrm{T}_{-667}, \mathrm{~T}_{-182}, \mathrm{~T}_{0}, \mathrm{~T}_{182}, \mathrm{~T}_{667}$ ] with randomized masker locations), and 10 tracks under the $\mathrm{M}_{\mathrm{V}} \mathrm{T}_{\mathrm{V}}$ condition in both Masker Fringe conditions. Because listeners had extensive experience with the $\mathrm{M}_{\mathrm{F}} \mathrm{T}_{\mathrm{F}}$ conditions from the preliminary experiment, they only received minimal additional training on the $\mathrm{M}_{\mathrm{F}} \mathrm{T}_{\mathrm{F}}$ conditions before the main experiment began (two tracks each of $\mathrm{M}_{0} \mathrm{~T}_{0}$ and $\mathrm{M}_{-667} \mathrm{~T}_{0}$ configurations under both Pulsed and Fringe condition).

\section{Results}

Average thresholds for the 8 conditions are shown in Figure 2. $\mathrm{M}_{\mathrm{F}} \mathrm{T}_{\mathrm{V}}, \mathrm{M}_{\mathrm{V}} \mathrm{T}_{\mathrm{F}}$, and $M_{V} T_{V}$ thresholds (in each Masker Fringe condition) are the average estimate of 20 thresholds (see Procedure subsection) for each listener and then averaged across listeners. The $\mathrm{M}_{\mathrm{F}} \mathrm{T}_{\mathrm{F}}$ thresholds shown are the weighted average of the estimated thresholds for the 169 masker/target configurations (i.e., weighted by the number of times each masker/target configuration was actually presented to each listener in $\mathrm{M}_{\mathrm{V}} \mathrm{T}_{\mathrm{V}}$ condition). The estimated thresholds were derived from the average of the two measured thresholds for each of the 12 selected configurations (the same 12 configurations that had been used 


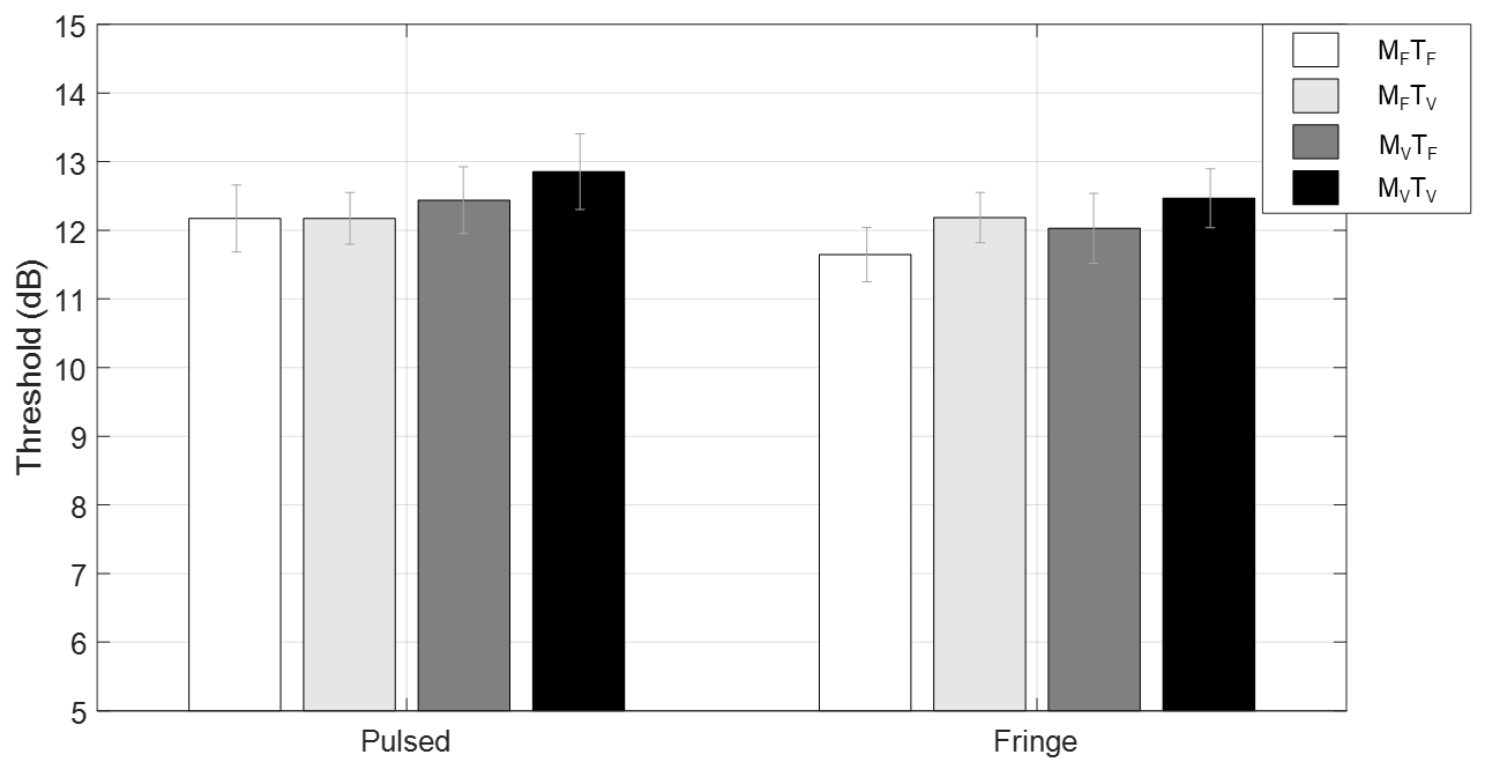

Figure 2. Threshold is plotted for the various spatial variability conditions in both Masker Fringe conditions. White bars represent the $\mathrm{M}_{\mathrm{F}} \mathrm{T}_{\mathrm{F}}$ condition, light grey bars represent the $\mathrm{M}_{\mathrm{F}} \mathrm{T}_{\mathrm{V}}$ condition, dark grey bars represent the $\mathrm{M}_{\mathrm{V}} \mathrm{T}_{\mathrm{F}}$ condition, and black bars represent the $\mathrm{MvT}_{\mathrm{V}}$ condition. Error bars show \pm 1.0 SE across subjects.

in the preliminary experiment), using procedures described in the Appendix. So, the $\mathrm{M}_{\mathrm{F}} \mathrm{T}_{\mathrm{F}}$ thresholds in Figure 2 are directly comparable to the $\mathrm{M}_{\mathrm{V}} \mathrm{T}_{\mathrm{V}}$ thresholds. Properly, $\mathrm{M}_{\mathrm{F}} \mathrm{T}_{\mathrm{F}}$ comparisons with other variable conditions $\left(\mathrm{MV}_{\mathrm{F}}\right.$ and $\mathrm{M}_{\mathrm{F}} \mathrm{T}_{\mathrm{V}}$ ) should also be weighted based on the configurations actually presented in those conditions (e.g., when comparing $\mathrm{M}_{\mathrm{F}} \mathrm{T}_{\mathrm{F}}$ and $\mathrm{M}_{\mathrm{V}} \mathrm{T}_{\mathrm{F}}$ conditions, threshold for $\mathrm{M}_{\mathrm{F}} \mathrm{T}_{\mathrm{F}}$ should reflect the average estimate of the 65 configurations used in the $\mathrm{Mv}_{\mathrm{F}}$ condition). In fact, this weighting made little difference (less than $0.1 \mathrm{~dB}$ ), so these bars are not shown.

The range of mean thresholds across the 8 conditions is $1.2 \mathrm{~dB}$. The results of a 3way repeated measures analysis of variance (ANOVA; Masker Fringe [pulsed, fringe] x 
Masker Variability [fixed, variable] x Target Variability [fixed, variable]) are summarized in Table 3. There was one significant main effect (Masker Variability) and no significant 2-way or 3-way interactions. Note, however, that despite the significant effect, the average difference between fixed and variable masker conditions is only about $0.4 \mathrm{~dB}$

Table 3

3-Way ANOVA Results for Target Variability, Masker Variability, and Masker Fringe

\begin{tabular}{|c|c|c|c|c|c|c|}
\hline & $\begin{array}{l}\text { Sum of } \\
\text { Squares }\end{array}$ & $d f$ & $\begin{array}{c}\text { Mean } \\
\text { Square }\end{array}$ & $F$ & $p$ & $\eta^{2} \mathrm{p}$ \\
\hline Fringe & 1.070 & 1 & 1.070 & 1.027 & .368 & .204 \\
\hline Error (Fringe) & 4.166 & 4 & 1.041 & & & \\
\hline Target Variability (TV) & 1.197 & 1 & 1.197 & 2.877 & .165 & .418 \\
\hline Error (TV) & 1.664 & 4 & .416 & & & \\
\hline Masker Variability (MV) & 1.592 & 1 & 1.592 & 77.465 & $.001^{*}$ & .951 \\
\hline Error (MV) & .082 & 4 & .021 & & & \\
\hline Fringe $x \mathrm{TV}$ & .195 & 1 & .195 & .961 & .382 & .194 \\
\hline Error (Fringe $x$ TV) & .811 & 4 & .203 & & & \\
\hline Fringe $\times \mathrm{MV}$ & .048 & 1 & .048 & .143 & .725 & .035 \\
\hline Error (Fringe x MV) & 1.347 & 4 & .337 & & & \\
\hline TV $x$ MV & .068 & 1 & .068 & .284 & .622 & .066 \\
\hline Error (TV x MV) & .959 & 4 & .240 & & & \\
\hline Fringe $\mathrm{x} T \mathrm{TV} \mathrm{xV}$ & .167 & 1 & .167 & 4.102 & .113 & .506 \\
\hline Error (Fringe $\mathrm{x}$ TV $\times$ MV) & .163 & 4 & .041 & & & \\
\hline
\end{tabular}

$* p<.05$

In the binaural detection literature investigating masker fringe (e.g., McFadden, 1966; Yost, 1985), both the location of the masker and the location of the target were fixed within each block of trials, and so, to compare our finding to that literature we focused on the $\mathrm{M}_{\mathrm{F}} \mathrm{T}_{\mathrm{F}}$ condition. These data are shown in Figure 3, where threshold 
(without the adjustment to equalize detectability) is plotted as a function of ITD difference (the difference between the masker ITD and the target ITD in $\mu$ s) for the pulsed and the fringe conditions. Note, because multiple spatial configurations have the same ITD difference (see Table 4) the plotted thresholds are averaged across those configurations, as well as across subjects. On average, threshold in the Pulsed condition was $4.6 \mathrm{~dB}$ higher than that in the fringe conditions, but the magnitude of this effect varied as a function of ITD difference.

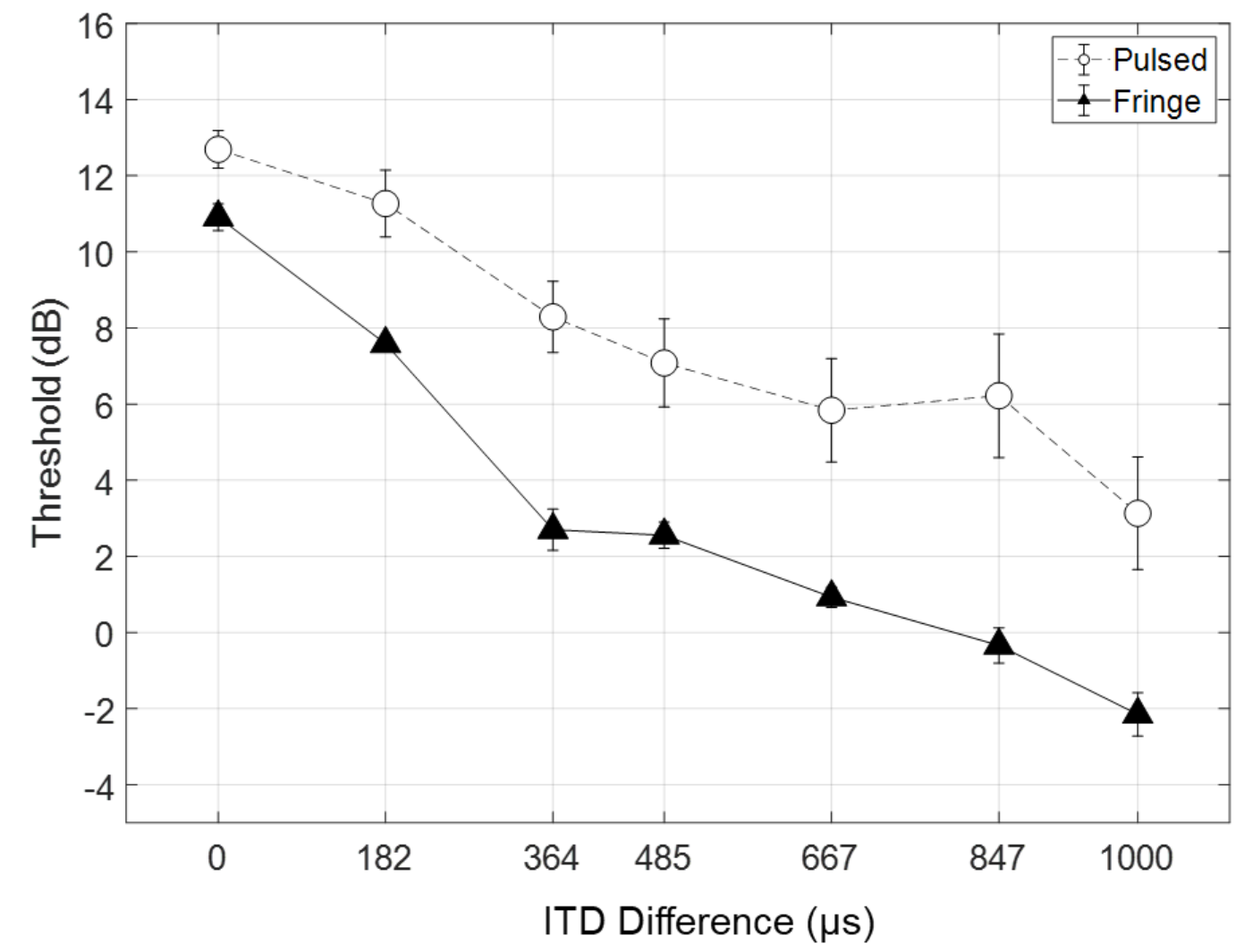

Figure 3. Threshold in the $\mathrm{M}_{\mathrm{F}} \mathrm{T}_{\mathrm{F}}$ condition is plotted as a function of ITD Difference (the difference between the masker ITD and the target ITD in $\mu \mathrm{s}$ ) in both Masker Fringe conditions. Triangles and solid lines represent the Fringe condition. Circles and dashed lines represent the Pulsed condition. Error bars show $\pm 1.0 \mathrm{SE}$ across listeners. 
Table 4

Differences in ITDs between masker and target for each $M_{F} T_{F}$ configuration

\begin{tabular}{|c|c|c|c|c|c|c|c|}
\hline $\begin{array}{l}\text { ITD Difference } \\
(\mu \mathrm{s})\end{array}$ & 0 & 182 & 364 & 485 & 667 & 847 & 1000 \\
\hline Configurations & $\begin{array}{c}\mathrm{M}-{ }_{667} \mathrm{~T}^{-}-667 \\
\mathrm{M}_{0} \mathrm{~T}_{0}\end{array}$ & $\begin{array}{l}M_{-182} T_{0} \\
M_{0} T_{182}\end{array}$ & $\mathrm{M}_{33} \mathrm{~T}_{-33}$ & $\begin{array}{l}M_{-667} T_{-182} \\
M_{-120} T_{-33}\end{array}$ & $\begin{array}{c}\mathrm{M}_{-667} \mathrm{~T}_{0} \\
\mathrm{M}_{0} \mathrm{~T}_{667} \\
\mathrm{M}_{-667} \mathrm{~T}_{667}\end{array}$ & $\begin{array}{l}\mathrm{M}_{-182} \mathrm{~T}_{667} \\
\mathrm{M}_{667} \mathrm{~T}_{-182}\end{array}$ & $\mathrm{M}_{0} \mathrm{~T}_{1000}$ \\
\hline
\end{tabular}

Note. Multiple configurations have the same ITD difference between masker and target.

We conducted a 2 x 7 (Masker Fringe [pulsed, fringe] x ITD Difference [0 $\mu$ s, $182 \mu \mathrm{s}, 364 \mu \mathrm{s}, 485 \mu \mathrm{s}, 667 \mu \mathrm{s}, 847 \mu \mathrm{s}, 1000 \mu \mathrm{s}])$ repeated measures ANOVA to examine the effects of Masker Fringe and ITD Differences on detectability. There was a significant main effect of Masker Fringe $\left(F(1,4)=30.22, p=.005, \eta_{\mathrm{P}}^{2}=0.88\right)$ indicating that thresholds were lower in the fringe condition, a significant main effect of ITD difference $\left(F(6,24)=76.50, p<.001, \eta_{\mathrm{P}}^{2}=0.95\right)$ indicating that thresholds generally decreased as ITD difference increased, as well as a significant interaction between Masker Fringe and ITD Difference $\left(F(6,24)=5.28, p=.001, \eta_{\mathrm{P}}^{2}=0.57\right)$ indicating that the onset effect varied significantly with ITD Difference. Note, the fact that we did not observe a signficant effect of Masker Fringe in the results shown in Figure 2 was partially due to the procedure used to equalize detectability. That is, this pocedure roughly normalized the thresholds for all masker/target spatial configrations to the threshold for the $\mathrm{M}_{0} \mathrm{~T}_{0}$ configuration. This was done seprately for Pulsed and Fringe conditions, so the overall difference between Pulsed and Fringe conditions should be equal to the difference 
for the $\mathrm{M}_{0} \mathrm{~T}_{0}$ configuration (about $1.0 \mathrm{~dB}$ ), unless there had been an interaction between ITD difference and Masker Variability and/or Target Vaiability.

We also conducted a 2 x 2 (Masker Fringe [pulsed, fringe] x Spatial Configuration $\left.\left[\mathrm{M}_{0} \mathrm{~T}_{0}, \mathrm{M}_{0} \mathrm{~T}_{1000}\right]\right)$ repeated measures ANOVA to specifically examine $\mathrm{N}_{0} \mathrm{~S}_{0}$ $\left(\mathrm{M}_{0} \mathrm{~T}_{0}\right)$ and $\mathrm{N}_{0} \mathrm{~S}_{\pi}\left(\mathrm{M}_{0} \mathrm{~T}_{1000}\right)$ configurations. These are the configurations that have most often been examined in binaural detection studies. There was a significant interaction between Masker Fringe and Spatial Configuration $\left(F[1,4]=8.45, p=0.04, \eta_{\mathrm{P}}^{2}=0.68\right)$. The effect of Masker Fringe (i.e., the difference in thresholds between the pulsed and the fringe conditions) was greater in the $\mathrm{N}_{0} \mathrm{~S}_{\pi}$ configuration relative to the $\mathrm{N}_{0} \mathrm{~S}_{0}$ configuration. Post hoc pairwise comparison analyses revealed a significant 5.3-dB onset effect under the $\mathrm{N}_{0} \mathrm{~S}_{\pi}$ configuration (Tukey HSD, $p<.05$ ), but an insignificant $1.0 \mathrm{~dB}$ onset effect $(p>.05)$ in the $\mathrm{N}_{0} \mathrm{~S}_{0}$ configuration. In addition, the MLD (i.e. the difference in masking between $\mathrm{N}_{0} \mathrm{~S}_{0}$ and $\mathrm{N}_{0} \mathrm{~S}_{\pi}$ configurations) was $4.3 \mathrm{~dB}$ greater in the Fringe condition than in the Pulsed condition $(p<.05)$.

\section{Discussion}

Our hypotheses about spatial variability and spatial cuing (in the $\mathrm{T}_{\mathrm{V}}$ conditions) were not supported by the results: 1) there was not a significant interaction between Masker Variability and Target Variability in the 3-way ANOVA, that is, there was little effect of Masker Variability in either the $\mathrm{T}_{\mathrm{F}}$ or $\mathrm{T}_{\mathrm{V}}$ conditions; 2) there was little spatial cuing effect, that is the there was little effect of masker fringe in either $\mathrm{M}_{\mathrm{F}}$ or $\mathrm{MV}_{\mathrm{V}}$ conditions and little effect of masker variability to mitigate; and 3) there was no 
interaction between Masker Fringe and Target variability in the 3-way ANOVA, that is, there was little effect of Masker Fringe in either the $T_{F}$ or $T_{V}$ conditions. However, when the $\mathrm{M}_{\mathrm{F}} \mathrm{T}_{\mathrm{F}}$ data were analyzed without the adjustment to equalize detectability, our hypotheses regarding the onset effect were supported: 1) there was a moderate onset effect comparable to observations in the detection literature and in Simpson's localization experiment; and 2) thresholds generally decreased as a function of ITD difference and the onset effect generally increased as a function of ITD differences.

\section{Effects of Spatial Variability}

Overall, we observed little effect of spatial variability on target detectability. The effect of Masker Variability was statistically significant, but only $0.4 \mathrm{~dB}$. The small effect of spatial variability we observed was of similar magnitude to the effects that others have reported for detection tasks (0.7-2.7 dB; Bernstein \& Trahiotis, 1997; Fan et al., 2008), but much smaller than what Simpson (2011) observed in a localization task (10.0 dB).

Arguably, there was substantially more spatial variability in the Simpson (2011) experiment than in any of the detection studies. Whereas in the current study and most studies in the binaural detection literature, the target stimuli were low-frequency, narrowband, and only varied in laterality (most often by varying ITD with headphone presentation), Simpson's stimuli were wideband and presented in the free field where they could vary in the Left/Right, Front/Back, and Up/Down dimensions. Simpson used over 15,000 potential masker/target configurations (239 masker locations by 64 target locations). Depending on condition, we used up to 169 masker/target configurations (13 
masker locations by 13 target locations). Fan et al. (2008) and Bernstein and Trahiotis (1997) each employed only 10 masker/target configurations. Because Simpson's localization task was conducted in the free-field and the locations of the sound sources varied in azimuth and in elevation, there were multiple spatial cues (ITDs, ILDs, monaural spectral cues, and binaural spectral cues), all with numerous possible values that varied randomly from trial to trial. Finally, recall that we equalized detectability across masker/target configurations. In Simpson's experiment, not only was there trial-totrial variation in multiple spatial cues, but substantial trial-to-trial variations in detectability, due not only to binaural interaction, but also to frequency dependent changes in monaural SNR because of head shadow and pinna effects (e.g., see Zurek, 1993).

Note, however, that Simpson (2011) observed the largest effect of spatial variability in the Left/Right dimension. Wightman and Kistler (1992) found that localization in the Left/Right dimension is dominated by ITDs in the low-frequency region, even when other conflicting cues were present. That is, our variations of low frequency ITDs should be representative of the major cue governing the Left/Right coordinate of Simpson's listeners' responses. And so, it seems unlikely that the much greater impact of masker spatial variability in Simpson's experiment can be attributed to the larger number of source locations and spatial cues varying in his experiment.

Bernstein and Trahiotis (1997) suggested that the effect of randomizing ITDs (i.e., spatial variability) was small in detection tasks because listeners based their decision on 
the width of the distribution of interaural parameters, not on its average value (e.g., the mean ITD). Recall in their study, target ITD and masker ITD were randomized together, and so, the width of the interaural distribution should stay about the same even as the average of the distribution changes from trial to trial. In the current study, we randomized the masker location and target location independently, that is, the width of the distribution varied from trial to trial. If listeners were basing their detection judgement on the width of the distribution of interaural parameters, we might expect to have observed an increased in the effect of spatial variability relative to Bernstein and Trahiotis; but, we did not.

Although the width of the distribution of interaural parameters seems like a viable cue in a binaural detection, it does not seem like a viable cue for localization. The Left/Right component of the localization judgement is more likely to be related to the mean of the distribution of interaural parameters. Introducing masker variability and/or target variability would lead to trial-to-trial variability in this mean value. And so, we would expect greater impact of spatial variability in a task based on the mean of the distribution of interaural parameters (localization or lateralization) than we would in a task based on the width of distribution of interaural parameters (detection).

Whatever the cues are that support localization and detection judgments, they are likely different. In Simpson's (2011) case the listeners had to choose the perceived source location from 239 loudspeaker locations. This spatial response requires processing and combining a number of different types of information (e.g., ITDs, ILDs, and spectral 
shape) in detail. The binary detection judgment is nonspatial and could rely on a single cue. In binaural conditions, this cue may (e.g., Hafter, 1971; Bernstein \& Trahiotis, 1997) or may not (e.g., Durlach, 1972; Gilkey, Robinson, \& Hanna, 1985) be particularly spatial in nature. So, perhaps it should not be surprising that the impact of spatial variability is different for a localization response than it is for a detection response

\section{Effect of the Masker Fringe}

As described in the Introduction, Simpson argued that there are two effects of the masker fringe: the spatial cuing effect and the onset effect. We observed little, if any, effect of masker variability, and so there was limited opportunity to observe a cuing effect. Moreover, because of the procedures we used to equalize detectability across masker/target configurations, the magnitude of any effect of masker fringe was forced to be small. That is, to equalize detectability, we normalized the level for all masker/target configurations to produce detectability equivalent to that expect for the $\mathrm{M}_{0} \mathrm{~T}_{0}$ configuration. This was done separately for pulsed and fringe conditions. And so, the overall difference we observed between the pulsed and the fringe conditions was about equal to the difference we observed between the pulsed and the fringe conditions when the masker/target configuration was diotic $\left(\mathrm{M}_{0} \mathrm{~T}_{0}\right)$ and fixed across trials.

However, the onset effect can be observed under the $\mathrm{M}_{\mathrm{F}} \mathrm{T}_{\mathrm{F}}$ condition if the procedures to equalize detectability are not applied. We observed that the difference between Pulsed and Fringe conditions generally increased as the difference between target and masker ITDs increased and was equal to $5.3 \mathrm{~dB}$ under the $\mathrm{M}_{0} \mathrm{~T}_{1000}\left(\mathrm{~N}_{0} \mathrm{~S}_{\pi}\right)$ 
condition, which is comparable to the values reported in the binaural detection literature (4.8-9.0 dB; McFadden, 1966; Robinson \& Trahiotis, 1972, \& Yost, 1985). Simpson reported an onset effect of $4.8 \mathrm{~dB}$, which was the difference in thresholds between pulsed and fringe conditions averaged across all masker/target ITD differences. We observed a 4.6-dB effect (averaged across the 12 measured masker/target configurations) for similar conditions.

Despite their similarity in magnitude, localization and detection onset effects may require different explanations. We consider three possible explanations for the onset effect: spatial transition, segregation, and overshoot.

To explain the substantially larger fringe effect under dichotic, as opposed to diotic conditions, McFadden (1966) argued that in the $\mathrm{N}_{0} \mathrm{~S}_{\pi}$ condition the masker fringe provides a diotic baseline that becomes dichotic when the target is added, changing the auditory image (e.g., causing it to move, widen, or shift its average position). He argued that this spatial transition provides an additional, more effective, cue for target detection that is not available in a diotic condition $\left(\mathrm{N}_{0} \mathrm{~S}_{0}\right)$. However, it is not clear whether such a spatial transition cue would be expected to be similarly useful in a localization task. If thought of as simply a detection cue, the argument becomes somewhat circular: changes in the spatial parameters of the stimulus lead to better detectability, better detectability leads to improved ability to hear out the spatial parameters of the target and thereby localize it. Moreover, whereas the transition McFadden described occurs when a diotic masker fringe transitions to a dichotic masker-plus-target, most of the maskers in 
Simpson's study were dichotic. So, in general, the transition will be from dichotic to dichotic. On the other hand, the spatial transition is not likely to be particularly reliable as a "direct" localization cue; any movement, widening, or change in the average position of the auditory image, could be toward or away from target, depending on the magnitude and phase of the noise component at the target frequency Although, the information in such a stimulus clearly forms the basis for the localization judgment, it is not immediately obvious that the transition per se is a particularly special or effective cue. Another possible explanation for the onset effect is sound source segregation. In natural situations, the various components of a new sound stimulus will typically begin together. However, the components of two different sound stimuli will typically begin at different times. So, two sounds with simultaneous temporal onsets are likely to be perceived as a single sound object coming from one source location. Two sounds with different onsets are likely to be segregated into two sound objects, which may come from different source locations (Bregman \& Pinker, 1978). Because the masker fringe separates the masker and target onsets temporally, they are more likely to be segregated into two objects that are separately localizable than would be the case in the Pulsed condition, in which the masker and target have the same onset. However, at low SNRs (detection studies) the sounds are unlikely to be segregated, and the target is "heard" as a change in the quality, spatial or otherwise, of the masker. So, segregation seems like a viable, if not particularly mechanistic, explanation for the onset effect in a localization task, but not in a detection task. 
The final explanation to consider is "overshoot" (Smith \& Zwislocki, 1975), which is typically used as an explanation for the increase in detectability (i.e., decrease in masking) observed in temporal masking experiments when the target onset is delayed relative to the masker onset. Although there is substantial neural activity at the onset of a new stimulus (e.g., the masker), this activity will decay over time. As a result, when a brief target is presented near the beginning of a long masker, the effective SNR is lower than when the same target is presented well after the masker onset. In the current experiment, the target onset is simultaneous with the masker onset in the Pulsed condition but is delayed in the Fringe condition. That is, the "masker fringe" should act to temporally separate the increase in neural activity associated with the target onset from the increase in neural activity associated with the masker onset, thereby increasing the effective SNR and allowing the target to be more readily detected. The effect in temporal masking experiments (i.e., the difference in threshold for brief targets near the beginning and end of a long masker) with a 500-Hz to1000-Hz target is about 3-5 dB (Zwicker, 1965; McFadden, 1988; Simpson, 1995), which overlaps with the range of fringe effects (about 5-9 dB) observed in the binaural detection literature (note, the condition in which a brief target was presented at the beginning of a long masker in a temporal masking experiment is more analogous to a backwards fringe condition. Trahiotis, Dolan, \& Miller, 1972, found lower threshold in the backwards fringe condition than in the Pulsed condition, but not as low as the forward fringe condition). 
It seems plausible that a model can be built using the neural explanation of overshoot that could be applied to explain both diotic and dichotic onset effects under both detection and localization settings. However, it is not completely clear how large the impact of overshoot is in dichotic masking conditions. Simpson (1995) observed a moderate dichotic overshoot effect (3.0 dB), whereas McFadden (1988) did not report any overshoot effect in the dichotic condition.

\section{Conclusion}

In summary, our results agree with previous findings in the literature and we were unable to resolve the discrepancy in the effects of spatial variability between localization and detection studies. We did not observe effects of masker spatial variability similar to those observed by Simpson (2011) even when target spatial variability was also present. Not surprisingly, given the negligible effect of masker variability, we did not find evidence for a spatial cuing effect of the masker fringe. However, we did observe onset effects similar to both Simpson and the binaural detection literature.

It is not immediately clear why we did not observe substantial effects of spatial variability. Changes in low frequency ITDs, which we focused on, are the dominant cues for localization in the Left/Right dimension, where Simpson (2011) observed the largest effects. Perhaps the effects of spatial variability are simply different for localization and detection tasks. That is, spatial variability may be less disruptive to cues for detection than cues for localization. It is also unclear whether the onset effects observed under 
detection and localization settings share the same explanation, but a model based on the neural explanation of overshoot at least seems plausible. 


\section{Reference}

Bernstein, L. R., \& Trahiotis, C. (1997). The effects of randomizing values of interaural disparities on binaural detection and on discrimination of interaural correlation, Journal of the Acoustical Society of America, 102, 1113-1120.

Fan, W. L., Streeter, T. M., \& Durlach, N. I. (2008). Effect of spatial uncertainty of masker on masked detection for nonspeech stimuli. Journal of the Acoustical Society of America, 124, 36-39.

Hafter, E. R. (1971). Quantitative Evaluation of a Lateralization Model of MaskingLevel Differences. The Journal of the Acoustical Society of America, 50, 11161122.

Gilkey, R. H., Robinson, D. E., \& Hanna, T. E. (1985). Effects of masker waveform and signal-to-masker phase relation on diotic and dichotic masking by reproducible noise. The Journal of the Acoustical Society of America, 78, 1207-1219.

Gilkey, R. H., Simpson, B. D., \& Wisenberger, J. M. (1990). Masker fringe and binaural detection. Journal of the Acoustical Society of America, 88, 1323-1332.

Kaernbach, C. (1990). A single-interval adjustment-matrix (SIAM) procedure for unbiased adaptive testing. Journal of the Acoustical Society of America, 88, 26452655.

McFadden, D. M. (1966). Masking-level differences with continuous and with burst masking noise. Journal of the Acoustical Society of America, 40, 1414-1419. 
McFadden, D. M. (1988). Absence of overshoot in a dichotic masking condition. Journal of the Acoustical Society of America, 83, 1685-1687.

Robinson, D. E., \& Trahiotis, C. (1972). Effects of signal duration and masker duration on detectability under diotic and dichotic listening conditions. Perception and Psychophysics, 12, 333-334.

Simpson, B. D. (1995). Evidence of overshoot in a dichotic masking condition. Unpublished manuscript, Central Insitute for the Deaf, Washington University, St. Louis, Missouri.

Simpson, B. D. (2011). Sound Localization in Multisource Environments: The Role of Stimulus Onset Asynchrony and Spatial Uncertainty (Doctoral dissertation). Retrieved from http://www.ohiolink.edu/etd/

Smith, R. L., \& Zwislocki, J. J. (1975). Short term adaptation and incremental responses of single auditory nerve fibers. Biological Cybernetics, 17, 169-182.

Spiegel, M. F., \& Green, D. M. (1981). Masker and signal uncertainty with noise maskers of varying duration, bandwidth, and center frequency. Journal of the Acoustical Society of America, 71, 1204-1210.

Yost, W. A. (1981). Lateral position of sinusoids presented with interaural intensive and temporal differences. Journal of the Acoustical Society of America, 70, 397-407.

Yost, W. A. (1985). Prior stimulation and the masking level difference. Journal of the Acoustical Society of America, 78, 901-907. 
Zurek, P.M. (1993), Binaural advantages and directional effects in speech intelligibility. In G.A. Studebaker and I. Hockberg (Ed.), Acoustical Factors Affecting Hearing Aid Performance (pp. 255-276). London, UK: Allyn and Bacon.

Zwicker, E. (1965). Temporal effects in simultaneous masking by white-noise bursts. Journal of the Acoustical Society of America, 37, 653-663 


\section{Appendix. Preliminary experiment}

In the $\mathrm{MVT}_{\mathrm{V}}, \mathrm{M}_{\mathrm{F}} \mathrm{T}_{\mathrm{V}}$, and $\mathrm{M}_{\mathrm{V}} \mathrm{T}_{\mathrm{V}}$ conditions of the main experiment, the spatial separation between the masker and the target varied randomly from trial to trial, and so, the detectability of the target also varied, due to the MLD. However, we were interested in the impact of spatial variability without the concomitant impact of trial-to-trial variations in detectability. Therefore, we conducted a preliminary experiment to estimate the target threshold (and MLD) for all spatial configurations for each listener. It was not practical to measure the target threshold for all 169 masker/target configurations (13 masker locations x 13 target locations) employed in this study. However, this number could easily be reduced to 25 by only making measurements for combinations of 5 locations $(1,4,7,10$, and 13 in Table 1$)$. These 25 configurations are equally spaced across the 13 x 13 set of possible masker/target configurations, such that all unmeasured masker/target configurations have target and masker locations that are within one spatial position of a measured location. The number of measured masker/target locations could be further reduced by noting that in 5 of the 25 configurations, the masker and the target are collocated and thus expected to have equal thresholds. Therefore, we only measured 2 of these collocated configurations. The remaining 20 locations were composed of 10 pairs of configurations that were symmetric about the median plane and thus expected to have equal thresholds. Therefore, we only measured one member of each pair.

Thresholds for the 12 measured configurations for one subject are shown in Figure A.1. Those measurements were used to estimate the MLD for all 169 spatial configurations 
under the Pulsed condition and under the Fringe condition. These estimates were in turn used in the main experiment to adjust the target level on each trial in order to maintain equal detectability across masker/target spatial configurations.

The same SIAM procedure as in the main experiment was used to obtain the 12 measured thresholds. The stimuli, trial structure, block structure, and session structure were the same as in the main experiment.

Because each of the 12 measured thresholds would be used to estimate the MLD for a number of other unmeasured spatial masker/target configurations (as described below), it was important that measurements were as accurate as possible. We initially conducted 3 adaptive tracks for each of the 12 spatial configurations. If the range of the 3 thresholds was greater than $3 \mathrm{~dB}$ for any configuration, we collected additional adaptive tracks for that configuration until there were at least three thresholds within $3 \mathrm{~dB}$ of each other. Most listeners were able to fulfill these requirements with 3-4 blocks for each configuration, no listener exceeded 6 blocks for any configuration. If the overall range of the 3-6 measured thresholds for any spatial configuration exceeded $5 \mathrm{~dB}$, we collected 2 additional tracks of data for that configuration. The overall threshold for each of the 12 masker/target configurations was calculated as the median of all threshold estimates for that configuration (3-8 tracks). Figure A.1 depicts the 169 masker/target configurations; the blackened cells show the 12 measured thresholds for one of the listeners under the Fringe condition. 
Figures A.2-A.5 illustrate the process of generating MLD estimates for the unmeasured masker/target spatial configurations. Note, the thresholds for the 12 measured configurations in Figure A.1 were converted to MLDs in Figures A.2-A.5. As stated, we assumed that configurations in which the masker and target were collocated would have equal thresholds. The average of the measured thresholds for the $\mathrm{M}_{-667} \mathrm{~T}_{-667}$ and $\mathrm{M}_{0} \mathrm{~T}_{0}$ was used as the threshold estimate for all configurations with collocated masker and target (blackened cells on the negative-sloped diagonal in Figure A.2). MLDs were calculated by subtracting the threshold of the collocated configurations from thresholds of each of the 12 measured configurations. Recall that we also assumed that configurations that were symmetric about the median plane would yield equivalent results, and so we used the MLDs from the measured configurations as the estimated MLDs for corresponding symmetric locations (blackened cells not on the negative-sloped diagonal line in Figure A.2).

The MLDs for the remaining 136 target/masker configurations were estimated by linearly interpolating between similar configurations for which the MLDs were known (i.e., previously measured or estimated). For example, MLDs for the blackened cells in Figure A.3 represent masker/target configurations that were estimated from two known configurations that shared the same target location and a masker location that differed by one or two spatial positions (or from known configurations that shared the same masker location and a target location that differed by one or two spatial positions). For example, to obtain the estimate for $\mathrm{M}_{-667} \mathrm{~T}_{-494}$, we took the weighted average of the MLDs for 
$\mathrm{M}_{-667} \mathrm{~T}_{-667}$ (weighed by 2.0) and $\mathrm{M}_{-667} \mathrm{~T}_{-182}$ (weighed by 1.0). MLDs for the blackened cells in Figure A.4 represent masker/target configurations that were estimated from four known surrounding configurations that shared the same target location and a masker location that differed by one spatial position (or from known configurations that shared the same masker location and a target location that differed by one spatial positions). For example, to obtain MLD estimate for M-494 $\mathrm{T}_{-309}$ we took the average estimate of $\mathbf{M}_{-667} \mathrm{~T}_{-309}, \mathbf{M}_{-494} \mathrm{~T}_{-494}, \mathbf{M}_{-309} \mathrm{~T}_{-309}$, and $\mathrm{M}_{-494} \mathrm{~T}_{-182}$. Finally, the blackened cells in Figure A.5 were calculated using weighted averages of the four surrounding configurations that shared the same target location and a masker location that differed by one or two spatial positions or shared the same masker location and a target location that differed by one or two spatial positions. For example, to obtain the MLD estimate for $\mathrm{M}_{-112} \mathrm{~T}_{-494}$, we took the weighted average of $\mathrm{M}_{-182} \mathrm{~T}_{-494}$ (weighed by 2.0), $\mathrm{M}_{-112} \mathrm{~T}_{-667}$ (weighed by 2.0), $\mathrm{M}_{0} \mathrm{~T}_{-494}$ (weighed by 1.0 ), and $\mathrm{M}_{-112} \mathrm{~T}_{-182}$ (weighed by 1.0 ). 


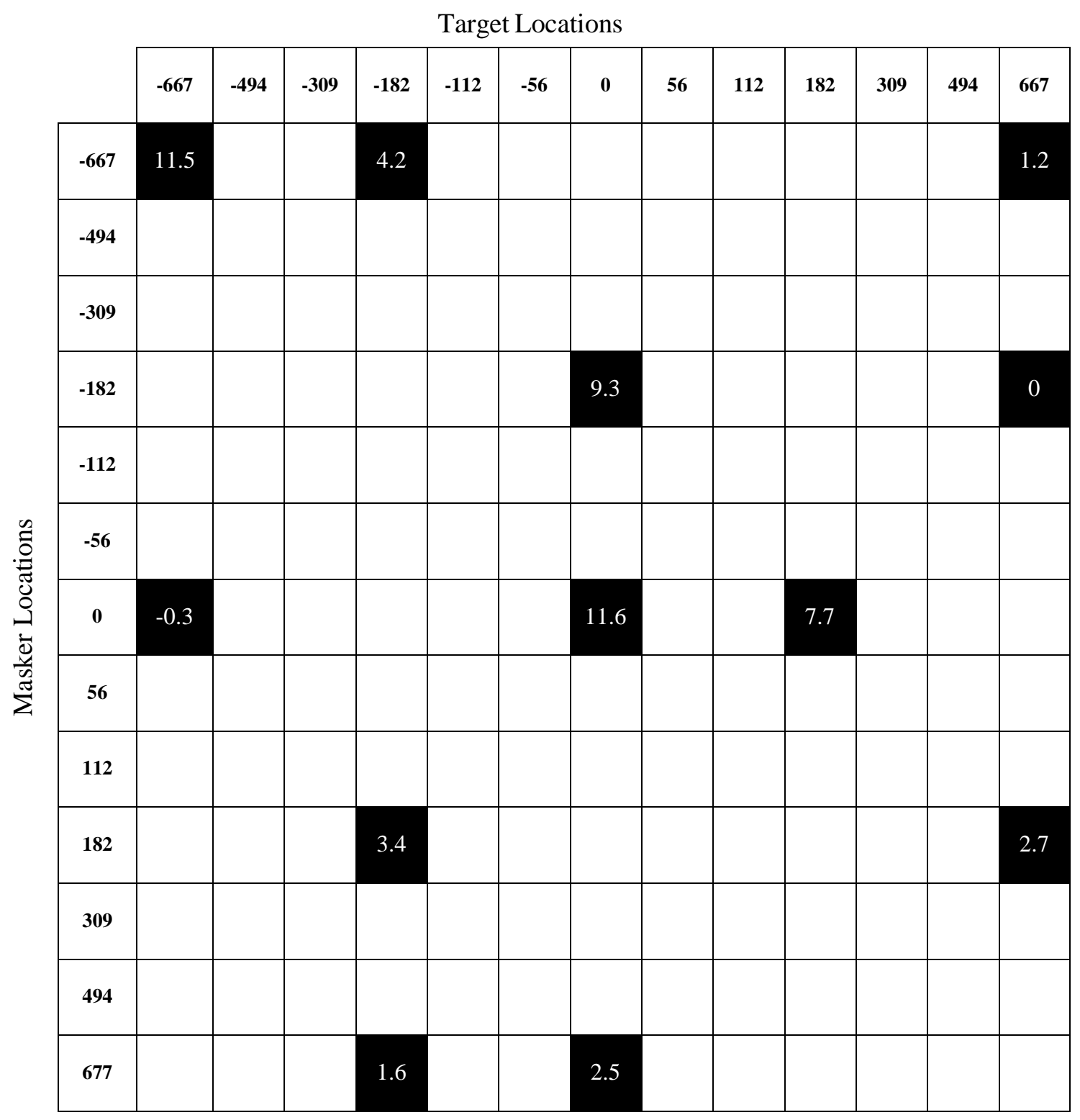

Figure A.1. Measured thresholds for 12 selected $\mathrm{M}_{\mathrm{F}} \mathrm{T}_{\mathrm{F}}$ configuration in the preliminary experiment. Data shown are for listener 1602 in the Fringe condition. Each row represents a masker location and each column represents a target location. Black cells represent measured configurations and the numbers displayed in the black cells are threshold estimates in $\mathrm{dB}$. 
Target Locations

\begin{tabular}{|c|c|c|c|c|c|c|c|c|c|c|c|c|c|}
\hline & -667 & -494 & -309 & -182 & -112 & -56 & o & 56 & 112 & 182 & 309 & 494 & 667 \\
\hline-667 & 0.0 & & & -7.4 & & & -9.1 & & & -10.0 & & & -10.3 \\
\hline-494 & & 0.0 & & & & & & & & & & & \\
\hline-309 & & & 0.0 & & & & & & & & & & \\
\hline-182 & -8.9 & & & 0.0 & & & -2.3 & & & -8.2 & & & -11.6 \\
\hline-112 & & & & & 0.0 & & & & & & & & \\
\hline-56 & & & & & & 0.0 & & & & & & & \\
\hline 0 & -11.9 & & & -3.9 & & & 0.0 & & & -3.9 & & & -11.9 \\
\hline 56 & & & & & & & & 0.0 & & & & & \\
\hline 112 & & & & & & & & & 0.0 & & & & \\
\hline 182 & -11.6 & & & -8.2 & & & -2.3 & & & 0.0 & & & -8.9 \\
\hline 309 & & & & & & & & & & & 0.0 & & \\
\hline 494 & & & & & & & & & & & & 0.0 & \\
\hline 677 & -10.3 & & & -10.0 & & & -9.1 & & & -7.4 & & & 0.0 \\
\hline
\end{tabular}

Figure A.2. MLD estimates for configurations in which the target and masker are collocated or that are symmetric about the median plane, to measured configurations (black cells). Grey cells represent MLDs for configurations that were shown in Figure A.1. The format of this figure is the same as Figure A.1. 
Target Locations

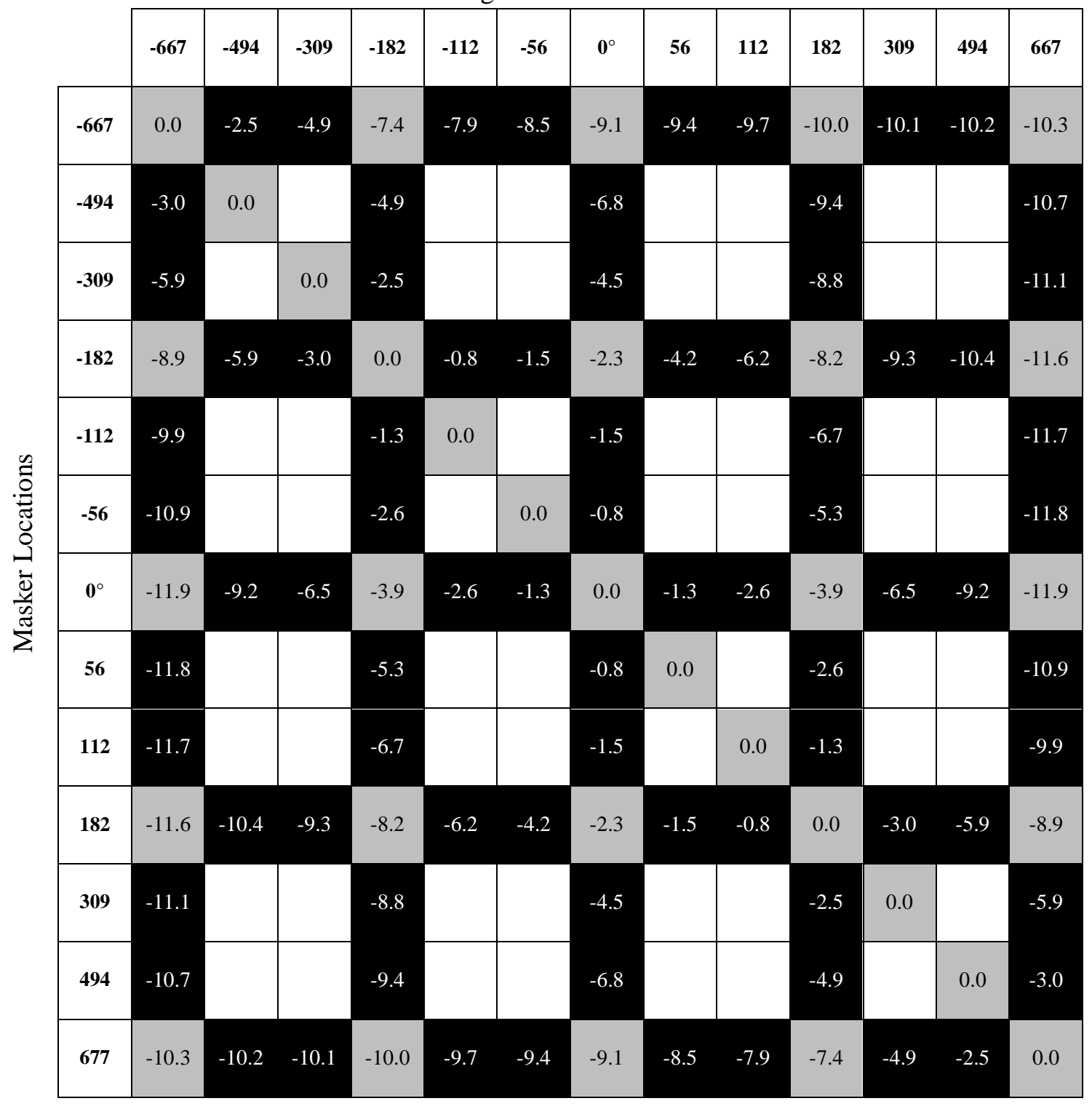

Figure A.3. MLD estimates calculated from two known masker/target configurations that share the same target location and a masker location that differed by two spatial positions or the same masker location and a target location that differed by two spatial positions (black cells). Grey cells represent MLDs that were shown in Figure A.2. The format of this figure is the same as previous figures in the Appendix. 
Target Locations

\begin{tabular}{|c|c|c|c|c|c|c|c|c|c|c|c|c|c|}
\hline & -667 & -494 & -309 & -182 & -112 & -56 & $\mathbf{0}^{\circ}$ & 56 & 112 & 182 & 309 & 494 & 667 \\
\hline-667 & 0.0 & -2.5 & -4.9 & -7.4 & -7.9 & -8.5 & -9.1 & -9.4 & -9.7 & -10.0 & -10.1 & -10.2 & -10.3 \\
\hline-494 & -3.0 & 0.0 & -2.5 & -4.9 & & & -6.8 & & & -9.4 & & & -10.7 \\
\hline-309 & -5.9 & -3.0 & 0.0 & -2.5 & & & -4.5 & & & -8.8 & & & -11.1 \\
\hline-182 & -8.9 & -5.9 & -3.0 & 0.0 & -0.8 & -1.5 & -2.3 & -4.2 & -6.2 & -8.2 & -9.3 & -10.4 & -11.6 \\
\hline-112 & -9.9 & & & -1.3 & 0.0 & -1.0 & -1.5 & & & -6.7 & & & -11.7 \\
\hline-56 & -10.9 & & & -2.6 & -1.3 & 0.0 & -0.8 & & & -5.3 & & & -11.8 \\
\hline $0^{\circ}$ & -11.9 & -9.2 & -6.5 & -3.9 & -2.6 & -1.3 & 0.0 & -1.3 & -2.6 & -3.9 & -6.5 & -9.2 & -11.9 \\
\hline 56 & -11.8 & & & -5.3 & & & -0.8 & 0.0 & -1.3 & -2.6 & & & -10.9 \\
\hline 112 & -11.7 & & & -6.7 & & & -1.5 & -0.8 & 0.0 & -1.3 & & & -9.9 \\
\hline 182 & -11.6 & -10.4 & -9.3 & -8.2 & -6.2 & -4.2 & -2.3 & -1.5 & -0.8 & 0.0 & -3.0 & -5.9 & -8.9 \\
\hline 309 & -11.1 & & & -8.8 & & & -4.5 & & & -2.5 & 0.0 & -3.0 & -5.9 \\
\hline 494 & -10.7 & & & -9.4 & & & -6.8 & & & -4.9 & -2.5 & 0.0 & -3.0 \\
\hline 677 & -10.3 & -10.2 & -10.1 & -10.0 & -9.7 & -9.4 & -9.1 & -8.5 & -7.9 & -7.4 & -4.9 & -2.5 & 0.0 \\
\hline
\end{tabular}

Figure A.4. MLD estimates calculated from four known masker/target configurations that shared the same target location and a masker location that differed by one spatial position or the same masker location and a target location that differed by one spatial position (black cells). Grey cells represent MLDs that were shown in Figure A.3. The format of this figure is the same as previous figures in the Appendix. 
Target Locations

\begin{tabular}{|c|c|c|c|c|c|c|c|c|c|c|c|c|c|}
\hline & -667 & -494 & -309 & -182 & -112 & -56 & $\mathbf{0}^{\circ}$ & 56 & 112 & 182 & 309 & 494 & 667 \\
\hline-667 & 0.0 & -2.5 & -4.9 & -7.4 & -7.9 & -8.5 & -9.1 & -9.4 & -9.7 & -10.0 & -10.1 & -10.2 & -10.3 \\
\hline-494 & -3.0 & 0.0 & -2.5 & -4.9 & -5.5 & -6.2 & -6.8 & -7.6 & -8.5 & -9.4 & -9.8 & -10.3 & -10.7 \\
\hline-309 & -5.9 & -3.0 & 0.0 & -2.5 & -3.1 & -3.8 & -4.5 & -5.9 & -7.3 & -8.8 & -9.5 & -10.3 & -11.1 \\
\hline-182 & -8.9 & -5.9 & -3.0 & 0.0 & -0.8 & -1.5 & -2.3 & -4.2 & -6.2 & -8.2 & -9.3 & -10.4 & -11.6 \\
\hline-112 & -9.9 & -7.0 & -4.1 & -1.3 & 0.0 & -1.0 & -1.5 & -3.2 & -5.0 & -6.7 & -8.4 & -10.0 & -11.7 \\
\hline-56 & -10.9 & -8.1 & -5.3 & -2.6 & -1.3 & 0.0 & -0.8 & -2.3 & -3.8 & -5.3 & -7.4 & -9.6 & -11.8 \\
\hline $0^{\circ}$ & -11.9 & -9.2 & -6.5 & -3.9 & -2.6 & -1.3 & 0.0 & -1.3 & -2.6 & -3.9 & -6.5 & -9.2 & -11.9 \\
\hline 56 & -11.8 & -9.6 & -7.4 & -5.3 & -3.8 & -2.3 & -0.8 & 0.0 & -1.3 & -2.6 & -5.3 & -8.1 & -10.9 \\
\hline 112 & -11.7 & -10.0 & -8.4 & -6.7 & -5.0 & -3.2 & -1.5 & -0.8 & 0.0 & -1.3 & -4.1 & -7.0 & -9.9 \\
\hline 182 & -11.6 & -10.4 & -9.3 & -8.2 & -6.2 & -4.2 & -2.3 & -1.5 & -0.8 & 0.0 & -3.0 & -5.9 & -8.9 \\
\hline 309 & -11.1 & -10.3 & -9.5 & -8.8 & -7.3 & -5.9 & -4.5 & -3.8 & -3.1 & -2.5 & 0.0 & -3.0 & -5.9 \\
\hline 494 & -10.7 & -10.3 & -9.8 & -9.4 & -8.5 & -7.6 & -6.8 & -6.2 & -5.5 & -4.9 & -2.5 & 0.0 & -3.0 \\
\hline 677 & -10.3 & -10.2 & -10.1 & -10.0 & -9.7 & -9.4 & -9.1 & -8.5 & -7.9 & -7.4 & -4.9 & -2.5 & 0.0 \\
\hline
\end{tabular}

Figure A.5. MLD estimates calculated from four known configurations that shared the same target location and a masker location that differed by one or two spatial position or the same masker location and a target location that differed by one or two spatial positions (black cells). Grey cells represent MLDs that were shown in Figures A.4. The format of this figure is the same as previous figures in the Appendix. 